\author{
(5)

\section{La inclusión educativa en una escuela normal rural: un estudio de caso} \\ Educational inclusion in a rural normal school: a case study \\ Inclusão educacional em escola normal rural: um estudo de caso
}

\author{
Ana Arán \\ Escuela Normal Rural Ricardo Flores Magón (México) \\ https://orcid.org/0000-0001-7149-3461
}

D0l: https://doi.org/10.35622/j.rep.2021.04.001

Recibido 02/12/2020/ Aceptado 16/11/2021

\begin{abstract}
RESUMEN. La concepción de la educación inclusiva forma parte del cambio paradigmático pedagógico de los últimos años, liderado por disposiciones legislativas a nivel mundial. Sin embargo, a nivel superior el acceso y permanencia a la educación para personas con discapacidad continúa siendo un reto. Se presenta una investigación cualitativa con metodología de estudio de caso, cuyo objetivo es conocer el proceso de inclusión educativa de una alumna con discapacidad motriz, estudiante en una escuela rural. Para tener un panorama integral del caso, se utilizó una encuesta sociodemográfica, entrevista estructurada del historial clínico, escala estimativa del INDEX de inclusión de Booth y Ainscow (2000) y una entrevista semi estructurada sobre la inclusión educativa. A través de la información obtenida, se evidencia la necesidad del fortalecimiento de las políticas a nivel superior, para eliminar las barreras de acceso y traslado, así como la implementación de una cultura inclusiva que erradique las prácticas discriminatórias.
\end{abstract}

PALABRAS CLAVE: Discapacidad, educación superior, estudio de caso, inclusión educativa.

ABSTRACT. The concept of inclusive education is part of the pedagogical paradigm shift in recent years, led by legislative provisions at the global level. However, access and permanence to education for people with disabilities continue to be a challenge at a higher level. Qualitative research with a case study methodology is presented, the objective of which is to know the process of educational inclusion of a student with motor disabilities, a student in a rural school. To have a comprehensive picture of the case, a sociodemographic survey, a structured interview of the clinical history, an estimation scale of the INDEX of inclusion by Booth and Ainscow (2000), and a semi-structured interview on educational inclusion were used. Through the information obtained, the need to strengthen policies at a higher level is evidenced to eliminate barriers to access and transfer and the implementation of an inclusive culture that eradicates discriminatory practices.

KEYWORDS: Disability, higher education, case study, educational inclusion.

RESUMO. 0 conceito de educação inclusiva faz parte da mudança de paradigma pedagógico nos últimos anos, liderada por disposições legislativas em nível global. No entanto, em um nível superior, 0 acesso e a permanência na educação para pessoas com deficiência continua sendo um desafio. É apresentada uma pesquisa qualitativa com metodologia de estudo de caso, cujo objetivo é conhecer o processo de inclusão educacional de um aluno com deficiência motora, aluno de uma escola rural. Para se ter uma visão abrangente do caso, foram utilizados um levantamento sociodemográfico, uma entrevista estruturada da história clínica, uma escala de estimativa do INDEX de inclusão de Booth e Ainscow (2000) e uma 
entrevista semiestruturada sobre inclusão educacional. Por meio das informações obtidas, evidencia-se a necessidade de fortalecimento das políticas em um nível superior, de eliminação de barreiras de acesso e transferência, bem como a implantação de uma cultura inclusiva que erradique práticas discriminatórias.

PALABRAS CLAVE: Deficiência, ensino superior, estudo de caso, inclusão educacional.

\section{INTRODUCCIÓN}

El término discapacidad continua siendo punto de debate, tanto en el ámbito académico, como el político y educativo (Pérez y Chhabra, 2019), por lo que ha experimentado una serie de modificaciones en cuanto a su conceptualización, pasando de describir este concepto en como una enfermedad a contextualizarlo en el enfoque de derechos (Hernández, 2015). En el 2006, la Asamblea General de las Naciones Unidas aprueba la Convención sobre los Derechos de las Personas con Discapacidad, para asegurar las condiciones de igualdad de derechos de todas las personas con discapacidad. Los estados ratificaron este documento, entre ellos México, están obligados a implementar y desarrollar políticas para evitar la discriminación de estos individuos y garantizar sus derechos. Implica un cambio de paradigma, ya que evoluciona de un marco asistencial a la exigencia de sus derechos y obligaciones como parte esencial de la sociedad. Un ejemplo de ello es el artículo 5 de esta convención, que establece la lgualdad y no discriminación, partiendo de la realización de los ajustes razonables para asegurar la igualdad de derechos, contemplando el contexto del individuo y no limitando el concepto de discapacidad a una condición intrínseca. Es por ello que autores como Guajardo (2017) afirman que "la discapacidad no es un asunto privado de las familias, sino que es una cuestión pública de la que los estados habrán de hacerse cargo porque rebasa con mucho la capacidad a la que puede ser capaz una familia" (p. 16).

En esta misma línea, el concepto de discapacidad actual se centra en la o las dificultades que un individuo experimenta para realizar una serie de actividades (INEGI, 2007), dejando a un lado definiciones anteriores que se centraban en la enfermedad de la persona y no en su funcionamiento: se va de lo intrínseco a lo extrínseco; y puede implicar dimensiones tanto psíquicas como físicas del individuo (Padilla-Muñoz, 2010). De acuerdo a Diniz, Barbosa y Dos Santos (2009) este término de alguna forma denuncia los ambientes que implican barreras y desigualdad para las personas con discapacidad. Así mismo, se identifica un considerable avance en este ámbito: "Actualmente se asume que una educación inclusiva supone entender que la escuela debe adaptarse a las necesidades de todo el alumnado, 
alejándose de una visión individual y exigiendo cambios en el sistema educativo" (Darretxe, Beloki y Remiro, 2020, p.78).

De acuerdo al Instituto Nacional de Estadística y Geografía (INEGI), en México, la cantidad de mujeres con discapacidad es superior a la de los hombres (3.8 millones frente a 3.3 millones en el último grupo). Adicionalmente, $6.2 \%$ de las mujeres del país tienen esta condición, que en el caso de los hombres se reduce al 5.7 por ciento. En el estado de Chihuahua, 66 de cada 1000 habitantes presentan algún tipo de discapacidad. Menciona también que el 59\% de las personas con discapacidad en México presenta dificultad para caminar y moverse, por delante del aspecto visual con $27 \%$ y auditivo (12\%). Este porcentaje representa a más de dos millones de personas en el país y alrededor del $20 \%$ de esta cifra tiene un rango de edad en etapa escolar.

En la investigación que a continuación se presenta, se analiza el caso de una estudiante con discapacidad motriz, y su proceso de inclusión educativa en una Escuela Normal Rural, instituciones en el nivel superior de educación en México que son formadoras de docentes. Dados los cambios paradigmáticos en cuenta a la concepción de la discapacidad, así como los diferentes acuerdos y convenciones ratificados a nivel mundial para asegurar los derechos de este sector de la población, entre ellos el acceso y permanencia en la educación, se considera relevante analizar la realidad y factibilidad de la educación inclusiva a nivel universitario.

\subsection{La inclusión educativa en la educación superior}

Toscano et al., (2017) argumentan sobre la necesidad imperante de que las instituciones a nivel superior en México sean realmente incluyentes. En su análisis sobre este tema, parten de la equidad como principio para "generar las condiciones de igualdad para el ingreso, la permanencia y el egreso al entorno social y productivo de todos los estudiantes" (p. 36).

Consideran que el avance en materia educativa para las personas con discapacidad, el nivel universitario, ha sido mínimo en los últimos años ( a pesar de la aprobación de leyes en este ámbito) debido a que "las acciones realizadas por la mayoría de las IES, no han logrado un posicionamiento claro que defina y articule la atención a la discapacidad en las funciones 
sustantivas de la universidad: docencia, investigación extensión de los servicios; por el contrario, la mayoría de sus acciones son fragmentadas, aisladas y descontextualizadas de la política institucional" (p. 40). Es por ello que concluyen que la mayoría de las Instituciones de Educación Superior (IES), no están listas para atender adecuadamente la diversidad del alumnado universitario.

Un caso concreto de inclusión educativa en la universidad es planteado por Gallegos (2011) acerca de la Universidad Politécnica Salesiana en Ecuador, en cuyo estudio explica las acciones implementadas en esta institución, de tipo organizacional y académico. El primer aspecto se centró en la infraestructura, eliminando las barreras de acceso a través de rampas, pasamos y elevadores.

También llevó a cabo sesiones de sensibilización. En el segundo rubro, encontramos la capacitación a los docentes, el rediseño de los planes de estudio para contemplar un enfoque inclusivo, adaptaciones curriculares por parte de los docentes y de los materiales para contemplar el sistema Braille. La autora concluye que, gracias a estas medidas, el centro "ha facilitado el acceso, permanencia y egreso de las poblaciones en condición de vulnerabilidad, en particular de las personas con discapacidad, a través de los diferentes programas que ofrece" (p. 120).

Las instituciones formadoras de docentes juegan un papel importante a la hora de desarrollar escuelas inclusivas en México. En este sentido, Flores, García y Romero (2017) indagan sobre las prácticas inclusivas de los docentes en este tipo de instituciones, particularmente en la Escuela Normal del Estado de San Luís Potosí. Parten de la premisa de que los docentes son el modelo a seguir, en relación con las prácticas, estilos de enseñanza y estrategias que implementan con sus estudiantes. Encuentran áreas de oportunidad respecto a la inclusión en este nivel educativo, ya que "la formación inicial docente da más prioridad a lo disciplinar que a lo pedagógico" (p. 41).

En su estudio, logran identificar áreas por mejorar en su institución, referente a la cultura y las prácticas, así como la capacitación de docentes. A su vez, encuentran confusión entre los términos de integración e inclusión, tanto en los docentes como los alumnos. Por otro 
lado, identifican que los docentes de la institución que formaron parte del estudio, tienen altas prácticas inclusivas.

Para abordar la cuestión específica de las escuelas normales, se considera pertinente examinar el estudio de Covarrubias et al., (2017) en el cual exploran los valores inclusivos en la Escuela Normal del Estado Chihuahua. Para ello, realizan una investigación de corte cuantitativo, aplicando una escala Likert a los alumnos de la institución, para el objetivo de conocer las tres dimensiones de la inclusión: cultura, política y práctica.

Los investigadores encuentran diferencias significativas en las respuestas de los alumnos de acuerdo al semestre que cursan: los estudiantes de primer semestre valoran de manera positiva la cultura, práctica y política de la escuela respecto a la inclusión, mientras que los alumnos más avanzados (séptimo semestre) hacen una evaluación desfavorable. En el aspecto de la aceptación y respeto hacia los alumnos con discapacidad se da el mismo fenómeno.

Finalmente, Alcaín y Medina (2017) indagan también sobre la inclusión en las universidades, pero en el sistema educativo español, argumentado que: "la falta de formación e información en los agentes implicados, la falta de apoyo y recursos educativos y de normas universitarias actualizadas nos sitúan ante unos retos que hay que abordar transversalmente para lograr la educación universitaria inclusiva real" (p. 17). Concluyen que se deben hacer esfuerzos para formar a los catedráticos universitarios en la temática de la inclusión y diseño universal.

\subsection{Fundamentación Teórica}

\section{a) Conceptos relacionados con la Educación Inclusiva}

En el ámbito de la educación de los individuos con discapacidad, se ha transitado por cuatro enfoques hasta ahora: de la exclusión se pasó a la educación especial, continuando con la integración, para finalmente llegar a la inclusión (Parra, 2010). En las últimas décadas, ha existido un cambio de actitud hacia las personas con discapacidad, reconociendo no solo sus derechos, sino también adoptando un rol activo como sociedad a la hora de sensibilizarse sobre su obligación de proveer igualdad de oportunidades para estas personas (Fedulova, Ivaniva, Atyukova y Nosov, 2019). Este cambio ha favorecido que se 
dejen atrás términos como "inválido" o "discapacitado", y que la definición tradicional de la discapacidad desde el enfoque del problema que caracteriza a la persona, evolucione al concepto de "persona con discapacidad", ya que en el centro está la persona (Dubkovetska, Budnyk y Sudoriv, 2016). De esta manera, se sustituye el concepto de deficiencia en los seres humanos, como lo establecía en modelo médico, y se avanza a un enfoque que hace énfasis en sus habilidades (Aust, 2018). Adicionalmente, mencionar que la discapacidad puede darse tanto en comunidades vulnerables con nutrición deficiente y dificultades para accesar los servicios de salud, como en contextos con facilidades en estos ámbitos (Ajisuksmo, 2017).

La educación inclusiva es un cambio global de sistema educativo (Mutaner et al., 2016) que implica "la accesibilidad a la educación para todas las categorías de los niños, en términos de adaptarse a las diversas necesidades de estos niños, buscando la forma óptima para su educación" (Fedulova et. al, 2019, p. 29). Por lo tanto, busca dar una respuesta de equidad (Echeita, 2017).

El enfoque en educación inclusiva involucra la creación de las condiciones para satisfacer las necesidades particulares que cada individuo presente, y la integración de las personas con discapacidad en su comunidad para que estudie, trabaje y tenga tiempo de ocio, es uno de los propósitos esenciales para implementar la educación inclusiva (Dubkovetska et al., 2016). En este sentido, la educación inclusiva busca eliminar la marginalización, aceptando las diferencias, y entendiendo que, sin importar estas, todos tenemos la oportunidad de ejercer un rol importante en nuestra sociedad (Ajisuksmo, 2017). De acuerdo a Fernández (2013) "el desarrollo de la inclusión educativa exige un análisis constante de las prácticas educativas y de los procesos de cambio escolar, no pudiendo reducirse simplemente a una ley" (p.83).

Por último, mencionar el concepto de integración, explicado como el proceso en el cual los alumnos con alguna discapacidad, no están todo el tiempo en el grupo "regular", sino que trabajan algunas horas aparte con un especialista con un curriculum adaptado (Varunek, 2020). De acuerdo a Fontana et al., (2009) esta "hace énfasis en el principio de normalización, sectorización e individualización" (p.22). Si se compara con la integración, el 
propósito de la inclusión es más amplio. Mientras que la primera aspira a "asegurar el derecho de las personas con discapacidad a educarse en las escuelas comunes, la inclusión aspira a hacer efectivo para toda la población el derecho a una educación de calidad" (Organización de las Naciones Unidas para la Educación, la Ciencia y la Cultura, 2008, p.7). Así mismo, mientras que el paradigma de la integración partía de la premisa de que los individuos tienen necesidades educativas especiales, el de la inclusión habla de las mismas pero debido al derecho de acceder a una educación que los incluya (Sinisi, 2010). Adicionalmente, se caracteriza por tener una visión centrada en la diversidad y la búsqueda para identificar y minimizar las barreras de acceso y permanencia.

\section{b) Marco Jurídico personas con discapacidad en México}

En el año 2011, la cámara de diputados publica la Ley General para la inclusión de las personas con discapacidad. Conceptualiza una serie de conceptos que resultan pertinentes para esta investigación. De acuerdo a esta ley, una persona con discapacidad es la que:

Por razón congénita o adquirida presenta una o más deficiencias de carácter físico, mental, intelectual o sensorial, ya sea permanente o temporal y que al interactuar con las barreras que le impone el entorno social, pueda impedir su inclusión plena y efectiva, en igualdad de condiciones con los demás (p.3).

El artículo cuatro de esta ley establece que todas las personas con discapacidad tienen los mismos derechos que las demás personas. Son merecedoras de acciones afirmativas positivas, como apoyos para disminuir su situación de desventaja o dificultad y establece también las medidas contra la discriminación hacia ellas.

Llama la atención que, en su definición del término educación inclusiva, habla de la integración de las personas con discapacidad únicamente en el nivel de educación básica. Sin embargo, en el tercer capítulo, dedicado a la educación, se establece en el artículo 12 y como segunda acción el "Impulsar la inclusión de las personas con discapacidad en todos los niveles del Sistema Educativo Nacional, desarrollando y aplicando normas y reglamentos que eviten su discriminación y las condiciones de accesibilidad en instalaciones educativas" (p. 8).

Por otro lado, en el 2014, como parte del Plan Nacional de Desarrollo 2013-2018, se publica 
el Programa Nacional Para El Desarrollo y La Inclusión De Las Personas Con Discapacidad 2014-2018. Este plantea una serie de objetivos que abarcan los derechos de las personas con discapacidad, su acceso a los servicios de salud, transporte y trabajo; así como su participación en diferentes ámbitos de la sociedad (cultural, deporte, tecnología, entre otras).

\section{c) El Índice de Inclusión}

La educación inclusiva es "un proceso orientado a responder a la diversidad del alumnado, incrementando su participación en la cultura, el currículo y las comunidades de las escuelas, reduciendo la exclusión en y desde la educación" (Marchesi et al., 2014, p.12). Además, implica una sociedad incluyente que, a través de la participación ciudadana, favorezca la inclusión de todos los individuos sin importar su contexto socioeconómico, étnico, religioso y de género, entre otros (Juárez et al., 2010). En este sentido, las escuelas formadoras de docentes tienen una gran responsabilidad para lograr que este tipo de educación sea una realidad en nuestro país, a través de la creación de una cultura, política y prácticas inclusivas (Booth y Ainscow, 2015).

La primera dimensión, la cultura, se centra en el fomento de los valores inclusivos, para que todos los agentes del proceso educativo sean valorados, y no solo respetados en sus diferencias, ya que enriquecen a la institución. Para ello, se sugiere la realización de talleres y actividades de sensibilización, con el objetivo de eliminar los prejuicios hacia lo "ajeno" o "diferente". En segundo lugar, tenemos a la dimensión política, la cual abarca el respeto a la diversidad, eliminando la discriminación, así como el acceso igualitario a una educación de calidad. Finalmente, las prácticas inclusivas implican eliminar las barreras hacia el aprendizaje y la participación del alumnado en actividades académicas, culturales y deportivas, entre otras.

Uno de los instrumentos utilizados en esta investigación está basado en el INDEX para la inclusión, creado por Booth y Ainscow en el año 2000 para evaluar a las escuelas en estas tres dimensiones de la educación inclusiva; un instrumento que permite ampliar el conocimiento sobre la inclusión en las instituciones educativas (Gutiérrez et al., 2014), a través de la evaluación de las tres dimensiones anteriormente mencionadas. Para ello, toma 
en cuenta las percepciones de los diferentes agentes que participan en el proceso pedagógico, como los docentes, estudiantes, familias y otros miembros del centro educativo (Booth et al., 2015). Además, esta herramienta ayuda a identificar las principales barreras para lograr una educación inclusiva: la presencia, el aprendizaje y la participación (Salceda e lbañez, 2015).

El Fondo de las Naciones Unidas para la Infancia (UNICEF) plantea a su vez una serie de indicadores para evaluar aspectos ambientales que afecten la participación en la escuela por parte de los infantes, ya que "Ios niños con discapacidad tienen menos probabilidades de ir a la escuela y más de abandonarla antes de completar un ciclo" (INEGI, 2017, p. 14). Si bien se centra en la infancia, puede servir como guía en la educación superior. Está formada por los dominios: actitudes, llegar a la escuela, accesibilidad dentro de la escuela y asequibilidad.

\subsection{Problematización}

En México existe un marco jurídico orientado a garantizar la educación de calidad a los estudiantes de todos los niveles educativos en nuestro país, sin importar su condición física, mental, racial y de género, a través, entre otros, la Ley General para la inclusión de las personas con discapacidad (2011) y el Programa Nacional Para El Desarrollo y La Inclusión De Las Personas Con Discapacidad 2014 así como en lo internacional la Convención sobre los Derechos de las Personas con Discapacidad (CDPCD) adoptada en México (2007) ; la realidad en las escuelas dista en gran medida de lo expuesto en estos lineamientos.

Si el ingreso a la educación básica para los niños con discapacidad se dificulta por las barreras de acceso, por ejemplo, los estudios a nivel superior representan un objetivo casi inalcanzable para muchos, "es evidente la poca participación de las personas con discapacidad en espacios escolarizados, se hace visible el grado de discriminación en que se encuentran algunos colectivos hoy en día" (Cruz, 2016, p. 4). Así mismo, el hecho de que un joven con discapacidad acceda a la educación universitaria, implica que ha tenido que "superar todo un proceso educativo y de escolarización no exento de dificultades, presentes desde sus primeros años, y que habrán podido salvar gracias a los recursos y apoyos educativos recibidos durante estas etapas precedentes" (Peralta, 2007, p. 13). 
En lo que se refiere a las instituciones formadoras de docentes, en las últimas reformas y planes de estudio (2018) que se han establecido han postulado cambios a nivel curricular en los planes y programas de estudio, contemplando asignaturas como Atención a la Diversidad y Educación Inclusiva, e incluso reestructurando la licenciatura de Educación Especial y renombrándola como licenciatura en Inclusión Educativa. A su vez, la Estrategia Nacional para el Fortalecimiento de las Escuelas Normales (2018), se plantean una serie de acciones de política pública, algunas de ellas encaminadas a la inclusión educativa, como "fortalecer la formación inicial docente para que los futuros maestros cuenten con las herramientas y estrategias para atender a las personas con discapacidad, con dificultades severas, de aprendizaje o de comunicación, así como de aquellas con aptitudes sobresalientes" (p. 26).

Sin embargo, se observa un vacío legislativo y que se traduzca en acciones concretas para la inclusión de estudiantes con alguna discapacidad en la educación universitaria, y que apoye su permanencia y posterior egreso. Consecuentemente, el propósito de esta investigación es conocer cómo es el proceso de inclusión educativa para una alumna con discapacidad que estudia en una escuela normal rural. Por lo tanto, se plantea la siguiente pregunta científica: ¿Cómo es el proceso de inclusión educativa para una alumna con discapacidad en una escuela normal rural?

\section{MÉTODO Y MATERIALES}

Esta investigación se enmarca dentro del paradigma interpretativo, ya que permite "el análisis de los significados de las acciones humanas, en su entorno histórico, social y cultural" (Burgo et al., 2019, p.324), analizando determinado fenómeno educativo a través de sus significados, símbolos e interpretaciones (Sáez, 1989). Su enfoque es cualitativo, pues se propone estudiar fenómenos particulares y no generalizables (Gil et al., 2017), dentro de su ambiente natural (Ramos, 2015). No busca generalizar sino describir un fenómeno particular de acuerdo a como los protagonistas de este lo perciben (Bonilla y Rodríguez, 1995). Su metodología es estudio de caso, partiendo de este como una "descripción detallada del caso, un análisis de los temas o asuntos y las interpretaciones o afirmaciones del investigador sobre el caso" (McMillan y Schumacher 2005, p. 45). Se logra 
recolectando datos de manera detallada y profunda, a través de diferentes fuentes (Creswell, 2017).

De manera particular, el estudio de caso de esta investigación se encuentra dentro de la categoría de estudios biográficos (Díaz et al., 2011). Se decidió utilizar la metodología del estudio de caso, ya que presentaba importantes ventajas para estudiar este fenómeno. En primer lugar, propicia un análisis profundo a través de la obtención de una cantidad importante de datos e información. También permitía el uso de diferentes instrumentos para complementar la información. Finalmente, resultó una forma óptima de obtener una visión integral del fenómeno de la inclusión educativa en el nivel de educación superior.

Adicionalmente, mencionar la escasez de estudios en Latinoamérica sobre la inclusión educativa en la educación superior qué utilizan la metodología de estudio de caso, ya que en su mayoría son estudios a gran escala que abarcan una gran cantidad de población (Clavijo et al., 2020; Cáceres et al., 2019; Soto et al., 2018; Lastre et al., 2019) que si bien ofrecen información relevante sobre el tema, carecen de las ventajas que el método de estudio único de caso tiene, al profundizar en la problemática estudiada a través de un único informante.

\subsection{Instrumentos}

Como instrumento principal, se utilizó escala estimativa (ver Anexo 1), basada en el INDEX de inclusión de Booth y Ainscow (2000) y adaptada para tratar específicamente los aspectos de Cultura, dimensiones de construir comunidad y establecer valores inclusivos, y Política, sobre el apartado de desarrollar una escuela para todos. Posteriormente, se utilizó una entrevista semi estructurada para ahondar sobre los temas planteados en la escala, a modo de conversación con la participante (Díaz et al., 2013) para conocer su perspectiva sobre los aspectos a los que había otorgado una calificación menor y el porqué de esa evaluación. De acuerdo con Cortés e Iglesias (2004), es un instrumento que permite recopilar información de diferentes aspectos sobre la problemática que se está investigando.

\subsection{Contexto de la Investigación}

Para salvaguardar la privacidad de la alumna, se utilizará un seudónimo para referirse a ella. Yoltzin presenta una discapacidad de física, entendida esta como "la secuela de una 
afección en cualquier órgano o sistema corporal" (Comisión de Política Gubernamental en Materia de Derechos Humanos, s/f, p. 12). Esta es de tipo motriz y transitoria, derivada de una serie de padecimientos. A pesar de que ella experimenta actualmente problemas de memoria a corto y a largo plazo, derivados de una complicación en una intervención que le realizaron para atender su padecimiento primario, no se considera que esta se pueda clasificar como discapacidad mental o intelectual, ya que no apareció antes de los 18 años (requisito para la primera categoría) ni "que detone una situación alterada de la realidad" (Ibídem), condición para la segunda.

Su largo proceso de diagnóstico comienza en la adolescencia, cuando le detectan desgaste en los nervios de extremidades. Seguidamente, le diagnostican con Guillan Barré. Cuando entra a la Escuela Normal Rural y sufre una crisis en la que pierde movilidad y sensibilidad en sus extremidades, le detectan una lesión en el bulbo raquídeo y mielitis transversa consecuencia de una segunda recaída de Guillian Barré. Se da de baja en la escuela, regresa al año siguiente, y de nuevo tiene problemas de movilidad, por lo que utiliza un andador. Más adelante encuentran un absceso en la cabeza que oprime su cerebro, causante de dolor de cabeza y mareos, por lo que la operan para retirárselo. Durante la intervención, convulsiona, como consecuencia comienza con lagunas mentales y experimenta dificultades en la memoria a corto y largo plazo.

Actualmente, estudia la licenciatura en educación primaria en una Escuela Normal Rural, en una ciudad del estado de Chihuahua. Actualmente se encuentra terminando el sexto semestre, lo que equivale al tercer año de los cuatro que dura la licenciatura. Las Escuelas Normales Rurales, son instituciones formadoras de maestros, creadas después de la Revolución Mexicana, y las primeras de este tipo en Latinoamérica (Salas, 2018). Como tal, sus ideales políticos se basaban en erradicar la pobreza a través de la educación. Por ello funcionan como internados, con el propósito de erradicar la desigualdad, cubriendo las necesidades de alimentación, uniforme, material didáctico, servicios médicos y asistenciales de los estudiantes. Resulta relevante notar que ella estaba residiendo en la institución, pero debido a diferentes crisis de salud derivadas de su padecimiento, tuvo que hacerse externa, ya que la infraestructura no le permitía realizar sus actividades cotidianas y académicas. 
Adicionalmente, notar que, en este tipo de instituciones de nivel superior, tienen cinco ejes en su proyecto de formación integral: el académico, el de producción agropecuaria, el eje cultural, el deportivo y el político a cargo de la FECSM (Federación de Estudiantes Campesinos Socialistas de México) (Coll, 2015). Lo anterior implica una serie de deberes y obligaciones de los estudiantes más allá de lo meramente académico como en otras instituciones formadores de docentes, mismas que deben de cumplir como parte de su formación integral. En el caso de la alumna protagonista de este estudio de caso y por su discapacidad motriz, el llevar a cabo algunas de estas tareas resulta complejo, dado las instalaciones del centro educativo y las barreras de acceso.

\section{RESULTADOS Y DISCUSIONES}

A continuación, se presentan los resultados de la aplicación de parte de la escala estimativa basada en el INDEX de inclusión de Booth y Ainscow (2000). Los datos obtenidos se organizaron en gráficas, comparando la puntuación asignada por Yoltzín en cada indicador con la puntuación ideal.

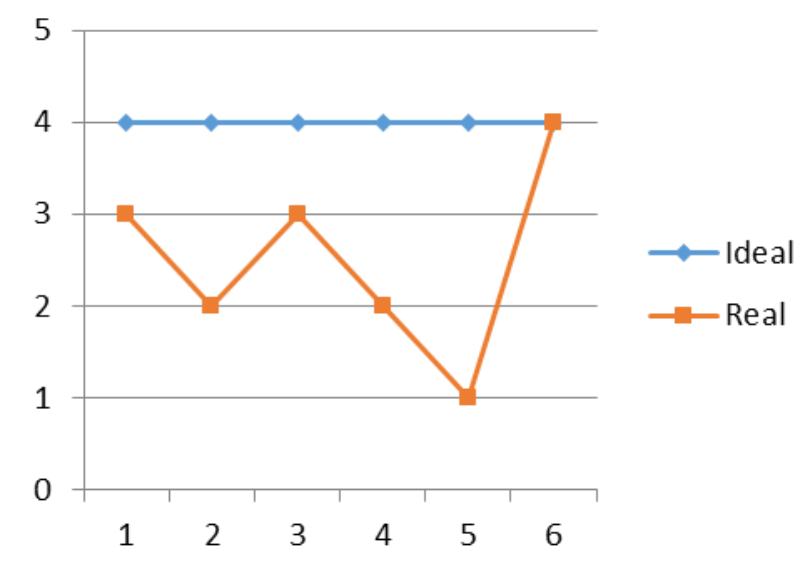

Figura 1. Cultura-Construir Comunidad

El indicador 2 "¿La escuela es acogedora para todos los estudiantes? es puntuado con una baja calificación. Yoltzin percibe falta de cultura hacia las personas con discapacidad en el entorno escolar. Como ejemplo explica que, si bien existen rampas, son utilizadas y obstruidas por alumnas que no las necesitan, molestándose cuando se les pide que muevan para poder pasar. El indicador n.5 (¿Has sido víctima de discriminación por tu discapacidad?) tiene una calificación inclusive más baja, lo cual resulta alarmante por el contenido que explora. Ella explica que las situaciones de discriminación se dan de manera 
frecuente en su grupo debido a la convivencia diaria. Por ejemplo, si van a salir a comer fuera de la escuela no la invitan, ya que consideran muy complicado transportarla en su silla de ruedas. Son frecuentes también los comentarios hirientes relativos a sus caídas frecuentes debido a su condición, como si los hiciera de manera intencional ("te tiras tú sola") y que muestran ignorancia y falta de empatía hacia su necesidad de la silla de ruedas para transportarse ("Tengo flojera para caminar, préstame tu carrito")

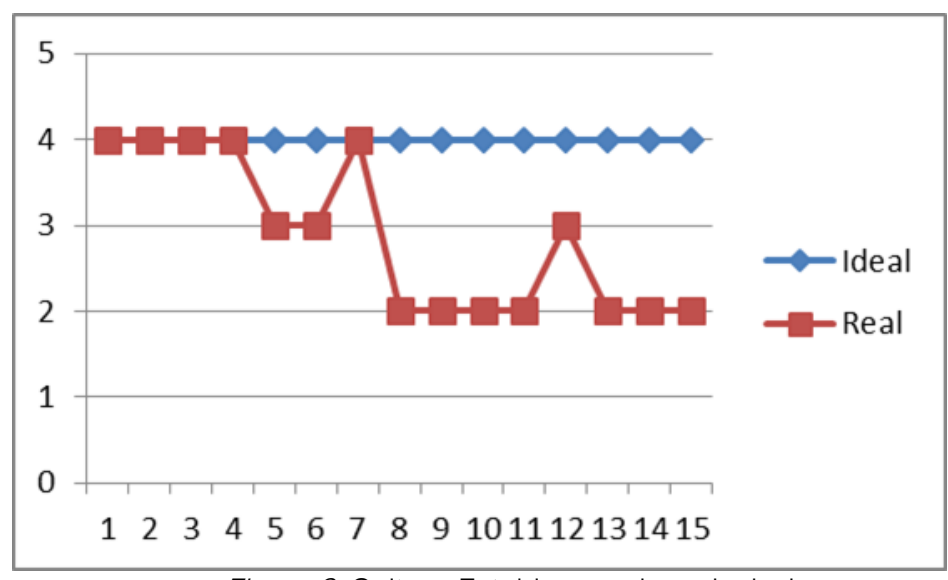

Figura 2. Cultura-Establecer valores inclusivos

En esta dimensión, Yoltzín califica de manera muy favorable la mayor parte de los primeros indicadores, relativos a los logros de aprendizaje de los estudiantes, la motivación y la valoración de estos. A partir del indicador 8 , sobre el uso de estereotipos y etiquetas, la puntuación baja drásticamente. Yoltzín ha vivido el uso de estereotipos tanto en la institución en la que estudia como en la escuela en la que practica. Algunos prejuicios que le ha escuchado sobre su persona $u$ otras estudiantes con alguna discapacidad es que son personas que no se peinan ni se bañan, y que por el hecho de que "sus piernas no sirven" no son capaces de hacer las actividades propias de un docente en formación, como elaborar material didáctico y diseñar planeaciones escolares. También considera que no se valora igual al alumnado con o sin discapacidad (indicador 9); suelen elegirla hasta el final para actividades en equipo de la clase de educación física o que requieren movilidad y en ocasiones se cuestionan las actividades que planea para las jornadas de práctica, considerando que no podrá realizarlas porque su discapacidad impide que tenga un control grupal adecuado.

Consecuentemente, Yoltzín considera que no se desafían las opiniones estereotipadas sobre la perfección física (indicador n. 13), ni las relacionadas con las personas con 
discapacidad son contrarrestadas por el personal de la institución (indicador n. 14). Si acaso, su situación acentúa la presión social al respecto, los comentarios como “¿Por qué no dejas de comer chucherías?" o "cuida lo que comes porque en la silla de ruedas usas menos energía" son frecuentes.

Considera que la institución implementa acciones para disminuir las prácticas discriminatorias (indicador 10), pero son superficiales e insuficientes, por lo que no se generan cambios. Por ejemplo, se imparten conferencias y talleres sobre la perspectiva de género, los derechos de las mujeres indígenas y derechos humanos; pero percibe desinterés por parte del alumnado para aprender sobre estos temas. También han proyectado películas sobre la discapacidad, pero no hay actividades posteriores de reflexión y análisis para promover la sensibilización del alumnado.

El caso de los maestros no es mucho más favorable, ya que no evitan ver la deficiencia como la única causa de las dificultades que experimentan en la escuela los estudiantes con discapacidad (indicador 11); siendo que Yoltzin afirma que, debido a su discapacidad física, algunos de ellos dudan de su capacidad intelectual y sus habilidades como futuro docente. Consecuentemente, la exclusión de los estudiantes con discapacidad se entiende como una dificultad práctica, y no como el reflejo de actitudes y políticas limitadas. A pesar de que su justificante médico la exenta de ciertas tareas, como alimentar a los animales, le asignan esa guardia como a cualquier otra alumna, teniendo que encontrar y en su caso pagar a alguien para que la cubra.

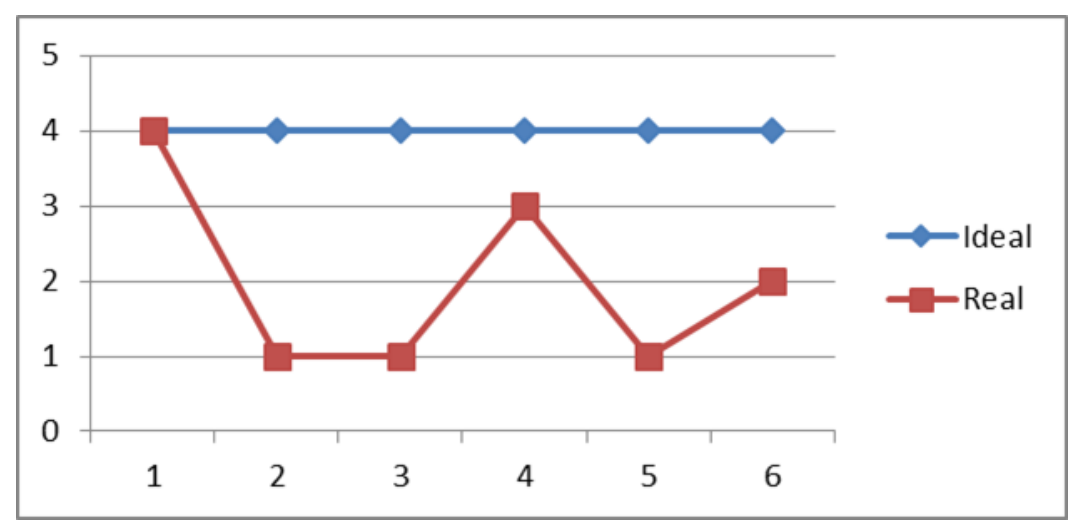

Figura 3. Políticas-Desarrollar una escuela para todos 
Yoltzín encuentra que, si bien ha aumentado la diversidad del alumnado (indicador n.1) pero que, lamentablemente, no se consideran sus necesidades para hacer los espacios de la escuela accesibles (indicador n. 2) En su caso, se construyó una rampa para que pudiera accesar a su salón de clases, pero esta no se diseñó correctamente y quedó un hueco en la parte de debajo de la puerta, permitiendo que entren animales y suciedad. Tampoco cree que se tomen en cuenta el acceso en el plan de mejora arquitectónico (n. 13), ya que para que una propuesta sea aceptada debe de pasar por muchos filtros y normalmente se llevan a cabo otro tipo de acciones.

Así mismo, observa falta de interés en la institución por conocer la legislación sobre la accesibilidad de las personas con discapacidad (indicador n. 5); sus compañeras no le preguntan las condiciones de la escuela en la que practica y en general no muestran ningún interés por su situación y los retos que esta implica, limitándose a acercarse a ella cuando necesitan algún apoyo de tipo académico (cabe resaltar que, a pesar de las faltas debido a su condición, Yoltzín es una alumna con un promedio de excelencia y con alto rendimiento académico) y la mayoría se queja cuando tienen que trabajar con estudiantes que tienen algún problema de aprendizaje.

Finalmente, sobre la accesibilidad de las diferentes instalaciones de la escuela (indicador $\mathrm{n}$. 6), como las aulas, baños y pasillos, Yoltzin tiene muchos ejemplos que compartir para ilustrar la baja calificación que le asignó a este indicador. Si bien hay rampas para accesar a diferentes espacios (no a todos, el gimnasio y el laboratorio de cómputo son inaccesibles para ella), estas no tienen la inclinación adecuada para garantizar una movilidad segura, están muy empinadas y llenas de baches. El pasillo para entrar al baño es tan estrecho que no le permite entrar con su silla de ruedas. El azulejo de las regaderas es muy resbaladizo y el espacio carece de barras de apoyo, por lo que bañarse no es seguro para ella. Debido a este tipo de barreras de acceso, Yoltzín tuvo que hacerse externa después de su última crisis.

Estas barreras de acceso y traslado se reflejan no sólo en la institución en la que Yoltzín estudia, sino en la escuela en la que practica. Una parte esencial de la formación de los futuros docentes es la práctica profesional; y en este semestre ella tuvo que cambiar de 
escuela de práctica, por la falta de transporte adecuado para ella y barreras de acceso. La opción más factible fue comenzar practicar en la escuela donde trabaja su madre, para que pudiera trasladarse con ella. En ese centro educativo facilitaron algunas medidas para eliminar las barreras de acceso, como construir una rampa para accesar a su salón de práctica y poner pasamanos en el baño (esta última medida fue antes de la segunda jornada de práctica, ya que previamente Yoltzín tenía que ser trasladada y apoyada por su madre). Ayudó el hecho de que es una escuela con recursos y que en el turno vespertino estudia una alumna con una discapacidad motriz.

\section{CONCLUSIONES}

Al analizar la información recabada, resulta evidente el extenuante, complejo y extenso proceso que Yoltzin ha experimentado en relación al diagnóstico y tratamiento de su condición. A pesar de tener la ventaja de contar con servicio de salud por sus padres, (se encuentra dentro del $52 \%$ de la población con discapacidad que cuenta con seguridad social y el 30\% que lo obtiene por familiares, de acuerdo al INEGI, 2017), ha tenido que pasar por una gran cantidad de especialistas para logar identificar su padecimiento, y encontrar un tratamiento adecuado; resulta evidente que "las personas con discapacidad son particularmente vulnerables a las deficiencias que presentan los servicios tales como la atención de salud, la rehabilitación y la asistencia y apoyo" (Organización Mundial de la Salud, 2011, p. 9)

La poca claridad y variedad de diagnósticos a lo largo de su padecimiento, le ha provocado conflictos con sus compañeras. Al principio no creían que tenía una enfermedad, que la eximiera de ciertas tareas propias de la organización escolar porque "no tenía un papel para demostrarlo". Además, su tipo de discapacidad implica otro reto en sí, ya que cuando se encuentra en una etapa relativamente estable la perciben como "igual” y le exigen de más, asignándole tareas que no puede lograr, y después aplicando consecuencias negativas como represalia, cuando se encuentra delicada le reclaman. Ha recaído en ella la tarea de sensibilizar e informar acerca de su enfermedad para que sus compañeras puedan comprender mejor su situación; es un proceso, pero considera que ha habido avances.

Con la información obtenida a través de la aplicación del instrumento, se evidencia los retos 
que Yoltzin vive diariamente, no debido a su discapacidad, sino a las condiciones del entorno. Por lo tanto, se concluye que los factores del entorno pueden resultar claves para el desarrollo pleno de los individuos.

La particularidad de que la institución en la que estudia tenga la modalidad de internado, representa una dificultad adicional, ya que no cuenta con la infraestructura adecuada para facilitar el traslado y el acceso a los diferentes espacios escolares dentro de ella, ni permite que ella cubra sus necesidades de higiene básicas. Lo cual está en consonancia con lo expresado por la Organización Mundial de la Salud (2011) en cuanto a la poca o nula accesibilidad a los edificios públicos y sistemas de transporte.

Cabe resaltar que ella estudia en esa institución porque en ella becan a las alumnas y le ofrecen diferentes servicios asistenciales como comedor, servicio médico, trabajo social y apoyo psicológico; y por la condición económica de su familia es el único tipo de formación universitaria que se pueden permitir. Aun así, ella se encuentra en el pequeño porcentaje de jóvenes con discapacidad que continúa sus estudios en el nivel de educación superior, solo el $14.6 \%$ de las mujeres con discapacidad asiste a la escuela en el rango de edad de 19 a 29 años, de acuerdo al INEGI (2017).

No solo la institución en la que estudia contiene barreras para ella, también la mayor parte de las escuelas de educación básica, por lo que el requisito de cumplir con las jornadas de práctica cada semestre se dificulta en gran medida. Desde el traslado al no existir transporte inclusivo que cuente con rampas hasta la estancia en la escuela, por el acceso al salón de clases, los baños etc.

Además de los obstáculos físicos a vencer, Yoltzín se enfrenta cada día con las actitudes y creencias del personal docente, de apoyo y alumnas de la institución. Lo anterior se refleja en poner en duda sus capacidades cognitivas, ser sujeta a comentarios despectivos, discriminatorios y excluyentes e incluso cuestionar que su problema motriz sea real. Resulta evidente que "las actitudes de los maestros, administradores escolares, otros niños e incluso familiares influyen en la inclusión de los niños con discapacidad en escuelas convencionales (p. 9)" Por si fuera poco, el hecho de ser mujer implica ser víctima por partida doble, ya que 
los estereotipos de género se añaden a las etiquetas con las que se discrimina a las personas con discapacidad.

Tomando en cuenta todos los retos con los que debe enfrentarse diariamente, no sorprende que, cuando se le pregunta de manera directa, Yoltzin concluya que la inclusión educativa no se puede lograr. Se inclina más por un modelo de integración, ya que considera que no puede realizar las mismas actividades que sus compañeras, debido a su estado de salud inestable y a las crisis continuas que experimenta. Pero el "problema" o la "dificultad motriz" no es la razón por la cual no puede realizar las mismas, son las barreras que existen en su contexto.

La movilidad es una de las actividades básicas de la vida cotidiana que refleja la interacción del organismo humano con el entorno; este tipo de dificultades están estrechamente relacionadas con la forma en la que está organizado el ambiente y si es accesible favorece la autonomía personal y la participación en ámbitos tan importantes como el educativo (INEGI, 2017, p. 48).

No hay que olvidar que la concepción sobre la discapacidad ha cambiado de manera radical en los últimos años, habiendo evolucionado de un concepto de "enfermedad, desorden o daño, una situación intrínseca" a "una condición de salud que incluye limitaciones en la actividad y restricciones en la participación. Indica aspectos negativos de la interacción entre un individuo y sus factores contextuales (ambientales y personales)" (p. 3). Por lo tanto, ella no es la que debe adaptarse al entorno, este debe ser lo suficientemente inclusivo para que pueda realizar las actividades que se requieren, así como cumplir con sus obligaciones.

Para comenzar a generar un cambio y lograr una verdadera educación inclusiva en el nivel superior, se deben tomar en cuenta la cultura, la política y las prácticas.

El éxito de los sistemas educativos inclusivos depende en gran medida del compromiso del país para adoptar una legislación apropiada, proporcionar una orientación normativa clara, elaborar un plan de acción nacional, establecer infraestructuras y crear capacidad de ejecución y asegurar una financiación a largo plazo (Organización Mundial de la Salud, 2011, p. 17). 
Sin olvidar los procesos de sensibilización para atender las creencias y actitudes que forman parte de la cultura, ya que "es fundamental mejorar la compresión pública de la discapacidad, oponerse a las percepciones negativas y representar la discapacidad, oponerse a las percepciones negativas y representar la discapacidad en su justa medida" (p. 21).

\section{REFERENCIAS BIBLIOGRÁFICAS}

Ajisujsmo, C. (2017). Practices and Challenges of Inclusive Education in Indonesian Higher Education. ASEACCU Conference on "Catholic Educational Institutions and Inclusive Education: Transforming Spaces, Promoting Practices, and Changing Minds". Assumption University of Thailand, Bangkok August 21-27, 2017.

Alcaín, E. y Medina-García, M. (2017). Hacia una educación universitaria inclusiva: realidad y retos. Revista Digital de Investigación en Docencia Universitaria, 11(1), 4-19. http://dx.doi.org/10.19083/ridu.11.530

Álvarez y San Fabián (2012). La elección del estudio de caso en investigación educativa. $\begin{array}{lllll}\text { Gazeta de } & 28 & \text { Antropología, } & \text { 1-12. }\end{array}$ http://www.ugr.es/ pwlac/G28_14Carmen_Alvarez-JoseLuis_SanFabian.pdf

Aust, R. (2018). Disability in Higher Education: Explanations and Legitimisation from Teachers at Leipzig University. Social Inclusion, 6(4), 125-136.

Booth, T., Simón, C., Sandoval, M., Echeita, G. y Muñoz, Y. (2015). Guía para la Educación Inclusiva. Promoviendo el Aprendizaje y la Participación en las Escuelas: Nueva Edición Revisada y Ampliada. REICE, 13(3), 5-19. http://www.redalyc.org/articulo.oa?id=55141402001

Bonilla-Castro, E. y Rodríguez, E. (1995). La investigación en las ciencias sociales. Más allá del dilema de los métodos. Norma: Bogotá.

Burgo, O.B., León, J.L., Cáceres, M.L., Pérez, C.J. y Espinoza, E.E. (2019). Algunas reflexiones sobre investigación e intervención educativa. Revista Cubana de Medicina Militar, 48(2), 316-330.___ http://scielo.sld.cu/scielo.php?pid=S0138$65572019000500003 \&$ script=sci_arttext\&tIng=en

Cáceres, C., Kaymalyz, C.I. y Ramírez, C. (2019). Índice de Inclusión Social en la Región de Valparaíso, Chile. Revista Mexicana de Sociología, 82(1), 93-122. http://mexicanadesociologia.unam.mx/index.php/v82n1/399-v82n1a4

Clavijo, R., Cabrera, F. y Japón, A. (2020). Evaluación de la aplicación del Índice de Inclusión en la Facultad de Psicología de la Universidad de Cuenca, Ecuador. Maskana, 11(1), 15-25. http://dx.doi.org/10.18537/mskn.11.01.02

Coll, T. (2015). Las Normales Rurales: noventa años de lucha y resistencia. El Cotidiano, 189, 83-94. https://biblat.unam.mx/es/revista/el-cotidiano/articulo/las-normalesrurales-noventa-anos-de-lucha-y-resistencia 
Comisión de Política Gubernamental en Materia de Derechos Humanos. (s/f). Glosario de Términos sobre

Discapacidad. http://www.semar.gob.mx/derechos_humanos/glosario_terminos_discapacidad.pdfs

Cortés, M. E. e Iglesias, M. (2004). Generalidades sobre Metodología de la Investigación. México: Universidad Autónoma del Carmen.

Creswell, J. (2017). Qualitative Inquiry and Research Design. California: SAGE.

Cruz, R. (2016). Discapacidad y educación superior: ¿Una cuestión de derechos o buenas voluntades? Revista de Investigación Educativa, 23, 1-23. http://www.scielo.org.mx/pdf/cpue/n23/1870-5308-cpue-23-00002.pdf

Darretxe, L., Beloki, N. y Remiro, A. (2020). Sociedad y escuela que deseamos: la dialéctica entre inclusión y exclusión social. Ciencia y Educación, (4)1, 71-81.: https://doi.org/10.22206/cyed.2020.v4i1.pp71-8

Díaz de Salas, Mendoza y Porras. (2011). Una guía para la elaboración de estudios de caso. Razón y Palabra, 16 (75). https://www.redalyc.org/articulo.oa?id=199518706040

Díaz-Bravo, L., Torruco-García, U., Martínez-Hernández, M. y Varela-Ruiz, M. (2013). La entrevista, recurso flexible y dinámico. Investigación en Educación Médica, 2(7), 162167.

http://www.scielo.org.mx/scielo.php?pid=S200750572013000300009\&script=sci_arttext

Diniz, D., Barbosa, L. y Dos Santos, W.r. (2019). Discapacidad, derechos humanos y justicia. Sur, 6(11), 65.77._https://www.scielo.br/scielo.php?pid=S1806$64452009000200004 \&$ script=sci_abstract\&tIng=es

Dubkovetska, I, Budnyk, O. y Sydoriv, S. (2016). Implementing inclusive education in Ukraine: problems and perspectives. Journal of Vasyl Stefanyk Precarpathian National University, 2(2-3), 99-105. http://dx.doi.org/10.15330/jpnu.3.2-3.99-105

Echeita, G. (2017). Educación Inclusiva. Sonrisas y Lágrimas. Aula Abierta, 46, 17-24. https://reunido.uniovi.es/index.php/AA/article/view/11982/11044

Fontana, A., Alvarado, A.L., Angulo, M., Marín, E. y Quirós D.M. (2009). El apoyo familiar en el proceso de integración educativa de estudiantes con necesidades educativas en condición de discapacidad. Revista Electrónica Educare, 13(2), 17-35. : http://www.redalyc.org/articulo.oa?id=194114401003

Fernández, J.M. (2013). Competencias docentes y educación inclusiva. Revista Electrónica de Investigación Educativa, 15(2), 82-99. http://redie.uabc.mx/vol15no2/contenidofdzbatanero.html

Flores, V.J., García, I. y Romero, S. (2017). Prácticas inclusivas en la formación docente en México. Liberabit, Revista de Psicología, 23(1), 39-56. https://doi.org/10.24265/liberabit.2017.v23n1.03

Gallegos, M. (2011). La inclusión educativa en la Universidad Politécnica Salesiana. Alteridad, Revista de Educación, 6(2), 118-126. https://alteridad.ups.edu.ec/index.php/alteridad/article/view/2.2011.03 
Gil, J.L., León, J.L. y Morales, M. (2917). Los paradigmas de investigación educativa, desde una perspectiva crítica. Revista Conrado, 13(58), $72-74$. http://conrado.ucf.edu.cu/index.php/conrado

Guajardo, E. (2017). La integración y la inclusión de alumnos con discapacidad en América Latina y el Caribe. Revista Latinoamericana de Educación Inclusiva, 3(1), 15-23.

Gutiérrez, M., Martín, V. y Jenaro, C. El Index para la inclusión: presencia, aprendizaje y participación. Revista Nacional e Internacional de Educación Inclusiva, 7(3), 186-201. https://gredos.usal.es/handle/10366/125635

Hernández, I. (2015). El concepto de discapacidad: de la enfermedad al enfoque de $\begin{array}{llll}\text { derechos. } & \text { Revista } & \text { CES } & \text { 46-59. }\end{array}$ https://dialnet.unirioja.es/servlet/articulo?codigo $=5280484$

Instituto Nacional de Estadística y Geografía, México. (2017). La discapacidad en México, datos al 2014. INEGI.

Juárez, J.M., Comboni, S. y Garnique, F. (2010). De la educación especial a la educación inclusiva.

Argumentos, 23(62),

41-83.

http://www.scielo.org.mx/scielo.php?pid=S018757952010000100003\&script=sci_a bstract\&tlng=en

Fedulova, I., Ivanova, I, Ivanova, V., Atyyukova, O. y Nosov, V. (2019). Inclusive Education as a Basis for Sustainable Development of Society. Journal of Social Studies

Education Research, (3),

118-135.

https://www.semanticscholar.org/paper/Inclusive-Education-as-a-Basis-for-

Sustainable-of-

Fedulovalvanova/2e5ab47d858ee17ec36b2794581b177186b95194

Lastre, K., Anaya, F. y Martínez, L. (2019). Índices de inclusión en una institución pública de Colombia. Revista $\quad$ Espacios, $40 \quad$ (33). http://www.revistaespacios.com/a19v40n33/19403316.html

Ley General para las personas con discapacidad. 30 de mayo del 2011. Diario Oficial de la Federación.

Marchesi, A., Blanco, R. y Hernández, L. (2014). Avances y desafíos de la educación inclusiva en Iberoamérica. Organización de Estados Iberoamericanos para la Educación, la Ciencia y la Cultura (OEI).

McMillan y Schumacher (2005). Investigación Educativa. Ciudad de México: Pearson

Mutaner, J.J., Roselló, M.R. y De la Iglesia (2016). Buenas prácticas en la educación Inclusiva. Education Siglo XXI, 34(1), 31-50. http://dx.doi.org/10.6018/j/252521

Organización de las Naciones Unidas para la Educación, la Ciencia y la Cultura (2008). La educación inclusiva: el camino hacia el futuro. Conferencia Internacional de Educación. Cuadragésima octava reunión, Centro Internacional de Conferencias, Ginebra.

Parra, C. (2010). Educación inclusiva: un modelo de educación para todos. Revista ISEES, $8,73-84$. 
Peralta, A. (2007). Libro Blanco sobre universidad y discapacidad. Real Patronato sobre Discapacidad, con la colaboración del Ministerio de Educación y Ciencia, la Fundación Vodafone, ANECA y el CERMI.

Pérez, M.E., y Chhabra, G. (2019). Modelos teóricos de discapacidad: un seguimiento del desarrollo histórico del concepto de discapacidad en las últimas cinco décadas. $\begin{array}{llll}\text { Revista Española de } & \text { 7-27. }\end{array}$ https://dialnet.unirioja.es/servlet/articulo?codigo=6955448

Salceda, M. e Ibáñez, A. (2015). Adaptación del Index for Inclusion al ámbito de la educación superior, estudio preliminar. Intangible capital, 11(3), 508-545. http://dx.doi.org/10.3926/ic.647

Navarro, E., Jiménez, E., Rappoport S. y Thoilliez, B. (2017). Fundamentos de la investigación y la innovación educativa. UNIR Editorial.

Padilla-Muñoz, A. (2015). Discapacidad: contexto, concepto y modelos. International Law: Revista Colombiana de Derecho Internacional, 16, 381-414. www.redalyc.org/articulo.oa?id=82420041012

Ramos, C.A. (2015). Los paradigmas de la investigación científica. Avances en Psicología, 23(1), 9-17. DOl: https://doi.org/10.33539/avpsicol.2015.v23n1.167

Saéz, J. (1989). El Enfoque interpretativo en Ciencias de la Educación. Anales de Pedagogía, 7, 7-32. https://revistas.um.es/analespedagogia/article/view/287761

Salas Marín, L. (2018). La política educativa del estado mexicano en relación a la formación inicial del profesorado de las escuelas normales rurales. Didácticas Especificas, (4), p. 77-95.

Sinisi, L. (2010). Integración o Inclusión escolar: ¿un cambio de paradigma?. Boletín de Antropología y Educación, 1, 11-14.

Soto-Cespedes, J.C. (2018). Índice de inclusión en una Institución Educativa de la ciudad de Cúcuta. Revista Científica Signos Fónicos, 4(1), 54-92. http://revistas.unipamplona.edu.co/ojs_viceinves/index.php/CDH/article/view/3945

Toscano, B.A., Ponce, J.C., Cruz, A., Zapien, A., Contreras, G. y Pérez, J.C. (2017). Análisis de la Inclusión en la Educación Superior en México. Una propuesta de Indicadores para los Organismos Acreditadores. Tecnología Educativa Revista CONAIC, IV (2), 35-51.

https://www.conaic.net/revista/publicaciones/Vol_IV_Num2_2017/Articulo_3.pdf

Varunek, V. (2020). Why Inclusion? Let's think together about what it means to have inclusion, and not just integration of children with special needs in regular schools. Electronic Research Journal of Social Sciences and Humanities, 2(1), 37-45 
ANEXO

Cuestionario para evaluar las escuelas inclusivas basado en INDEX de Inclusión

Booth y Ainscow (2000)

\begin{tabular}{|l|c|c|c|}
\hline $\begin{array}{l}\text { Completamente de } \\
\text { acuerdo }\end{array}$ & 2 & 3 & 4 \\
De acuerdo & En desacuerdo & $\begin{array}{l}\text { Completamente en } \\
\text { desacuerdo }\end{array}$ \\
\hline
\end{tabular}

\begin{tabular}{|c|c|c|}
\hline \multicolumn{3}{|c|}{$\begin{array}{l}\text { CULTURA } \\
\text { Construir una comunidad }\end{array}$} \\
\hline \multirow[t]{3}{*}{$\begin{array}{l}\text { Todo el mundo se } \\
\text { siente escogido }\end{array}$} & $\begin{array}{l}\text { ¿Es amistoso y acogedor el primer contacto que } \\
\text { tiene la gente con la escuela? }\end{array}$ & 1234 \\
\hline & $\begin{array}{l}\text { ¿La escuela es cogedora para los estudiantes con } \\
\text { discapacidad? }\end{array}$ & 1234 \\
\hline & $\begin{array}{l}\text { ¿Te sientes parte de tu clase? ¿De la academia de } \\
\text { segundo? }\end{array}$ & 1234 \\
\hline \multirow{2}{*}{$\begin{array}{l}\text { Los estudiantes se } \\
\text { ayudan unos a } \\
\text { otros }\end{array}$} & $\begin{array}{l}\text { ¿Te ayudan tus compañeras y maestras cuando lo } \\
\text { necesitas? }\end{array}$ & 1234 \\
\hline & $\begin{array}{l}\text { ¿Has sido víctima de discriminación por tu } \\
\text { discapacidad? }\end{array}$ & 1234 \\
\hline $\begin{array}{l}\text { El personal de la } \\
\text { escuela y el } \\
\text { alumnado se } \\
\text { tratan con } \\
\text { respeto. }\end{array}$ & ¿El personal se dirige contigo con respeto? & 1234 \\
\hline \multicolumn{3}{|c|}{ CULTURA: Establecer valores inclusivos } \\
\hline \multirow{5}{*}{$\begin{array}{l}\text { Se tienen altas } \\
\text { expectativas } \\
\text { respecto de todo } \\
\text { el alumnado. }\end{array}$} & $\begin{array}{l}\text { ¿Todo el alumnado siente que asiste a un centro } \\
\text { donde es posible que consiga sus mayores logros? }\end{array}$ & 1234 \\
\hline & $\begin{array}{l}\text { ¿Se motiva a todo el alumnado a que tenga } \\
\text { aspiraciones altas sobre su aprendizaje? }\end{array}$ & 1234 \\
\hline & $\begin{array}{l}\text { ¿Se trata de todo el alumnado como si sus logros no } \\
\text { tuvieran ningún tope? }\end{array}$ & 1234 \\
\hline & $\begin{array}{l}\text { ¿Se hace consciente al alumnado de que su éxito } \\
\text { depende de su esfuerzo? }\end{array}$ & 1234 \\
\hline & $\begin{array}{l}\text { ¿Se valora el logro del alumnado en relación con sus } \\
\text { propias posibilidades, en vez de en comparación con } \\
\text { el logro de los demás? }\end{array}$ & 1234 \\
\hline
\end{tabular}




\begin{tabular}{|c|c|c|}
\hline & $\begin{array}{l}\text { ¿Se motiva a todo el alumnado a que aprecie los } \\
\text { logros de los demás? }\end{array}$ & 1234 \\
\hline & $\begin{array}{l}\text { ¿Se motiva a todo el alumnado a que esté orgulloso } \\
\text { de sus propios logros? }\end{array}$ & 1234 \\
\hline & $\begin{array}{l}\text { ¿Se evita el uso de estereotipos de estudiantes a } \\
\text { través de etiquetas generales, especialmente del } \\
\text { alumnado con dificultades de aprendizaje o de otras } \\
\text { etnias, contextos y culturas? }\end{array}$ & 1234 \\
\hline $\begin{array}{l}\text { Se valora de igual } \\
\text { manera a todos } \\
\text { los alumnos y } \\
\text { alumnas. }\end{array}$ & $\begin{array}{l}\text { ¿Se valora de igual forma al alumnado y personal } \\
\text { con discapacidad y sin discapacidad? }\end{array}$ & 1234 \\
\hline $\begin{array}{l}\text { La escuela se } \\
\text { esfuerza en }\end{array}$ & $\begin{array}{l}\text { ¿Intenta la escuela disminuir toda discriminación } \\
\text { institucional, en relación con la discapacidad? }\end{array}$ & 1234 \\
\hline $\begin{array}{l}\text { prácticas } \\
\text { discriminatorias. }\end{array}$ & $\begin{array}{l}\text { ¿El profesorado evita ver la deficiencia como la única } \\
\text { causa de las dificultades que experimentan en la } \\
\text { escuela los estudiantes con discapacidad? }\end{array}$ & 1234 \\
\hline & $\begin{array}{l}\text { ¿El personal reconoce la limitada contribución que } \\
\text { ofrece el conocimiento de las discapacidades para } \\
\text { entender las posibilidades educativas de un } \\
\text { estudiante específico? }\end{array}$ & 1234 \\
\hline & $\begin{array}{l}\text { ¿Se desafían las opiniones estereotipadas sobre la } \\
\text { perfección física? }\end{array}$ & 1234 \\
\hline & $\begin{array}{l}\text { ¿Intenta el personal contrarrestar las actitudes } \\
\text { estereotipadas hacia las personas con discapacidad } \\
\text { (por ejemplo, que son incapaces de tener } \\
\text { relaciones, que merecen compasión o que son } \\
\text { luchadores heroicos contra la adversidad)? }\end{array}$ & 1234 \\
\hline & $\begin{array}{l}\text { ¿Se entiende la exclusión de los estudiantes con } \\
\text { discapacidades severas como el reflejo de las } \\
\text { limitaciones en las actitudes y las políticas, en vez de } \\
\text { cómo dificultades prácticas? }\end{array}$ & 1234 \\
\hline Desarrollar una esc & jela para todos & \\
\hline $\begin{array}{l}\text { La escuela intenta } \\
\text { admitir a todo el } \\
\text { alumnado de su } \\
\text { localidad. }\end{array}$ & $\begin{array}{l}\text { ¿Hay evidencia de un aumento de la diversidad del } \\
\text { alumnado de lo localidad que está incluido en la } \\
\text { escuela? }\end{array}$ & 1234 \\
\hline $\begin{array}{l}\text { La escuela hace } \\
\text { que sus } \\
\text { instalaciones sean } \\
\text { físicamente }\end{array}$ & $\begin{array}{l}\text { ¿Se consideran las necesidades de las personas con } \\
\text { discapacidades físicas, a la hora de hacer los } \\
\text { edificios y espacios de la escuela accesibles? }\end{array}$ & 1234 \\
\hline
\end{tabular}




\begin{tabular}{|c|c|c|}
\hline \multirow[t]{4}{*}{$\begin{array}{l}\text { accesibles para } \\
\text { todos. }\end{array}$} & $\begin{array}{l}\text { ¿Se considera el acceso de las personas con } \\
\text { discapacidad en el plan de mejoramiento } \\
\text { arquitectónico de la escuela? }\end{array}$ & 1234 \\
\hline & $\begin{array}{l}\text { ¿Se han tomado medidas para ir mejorando la } \\
\text { accesibilidad al centro y a sus dependencias como } \\
\text { parte del proyecto de la escuela? }\end{array}$ & 1234 \\
\hline & $\begin{array}{l}\text { ¿La escuela se preocupa por conocer la legislación } \\
\text { existente en el país en relación con la accesibilidad } \\
\text { de las personas con discapacidad? }\end{array}$ & 1234 \\
\hline & $\begin{array}{l}\text { ¿Hay una preocupación por la accesibilidad de los } \\
\text { edificios y las áreas del centro en todos los aspectos, } \\
\text { incluyendo las aulas, los pasillos, los baños, los } \\
\text { jardines, las áreas de juego, el comedor o cafetería } \\
\text { y las exposiciones? }\end{array}$ & 1234 \\
\hline
\end{tabular}

